\title{
Magnetic Field Based Positioning Systems
}

\author{
Valter Pasku, Alessio De Angelis, Guido De Angelis, Darmindra Arumugam, Marco Dionigi, Paolo Carbone, \\ Antonio Moschitta, David Ricketts
}

\begin{abstract}
This paper has the main purpose to give an introductory survey on the various systems that exploit magnetic fields for positioning. Such systems find applications in those scenarios, both indoors and outdoors, where Global Navigation Satellite Systems (GNSS) are not available or fail to provide information with the needed accuracy. While the main idea of using electromagnetic fields to provide position information dates back to the past century, new application-led research on this topic has emerged in recent years. Results have expanded the application range of Magnetic Positioning (MP) technologies and form now a domain of knowledge that enables realization of positioning systems applicable to indoor and outdoor environments.

The paper provides the main characteristics of different positioning systems with focus on those solutions that are based on low-frequency magnetic fields. Some background theory is presented and positioning results from the literature are analyzed and compared.
\end{abstract} GNSS, indoor positioning.

\section{INTRODUCTION}

$\mathbf{P}$ OSITIONING of users, devices, and systems has become very popular in the last decades. In fact, it acts as an enabler for different applications. Examples are several Location Based Services (LBSs) [1] $-[3]$, such as line traceability, industrial automation, assisted navigation of hospitals and public buildings, and operation under emergency conditions in hostile environments (i.e. firefighters). Moreover, location awareness is becoming an enabling technology for applications under the paradigm of the Internet of Things [4]-[6] and an essential part of everyday life under the Ambient Assisted Living (AAL) framework [7], [8].

In this context, information about location is beneficial for many environments. While satellite-based Global Navigation Systems (GNSs) are well established for use in outdoor environments, their applicability is low in indoor environments. Due to the structure of the indoor environment, the development of positioning systems is challenging. Moreover, system requirements are strongly affected by the specific application scenario. This has an impact on such parameters as target accuracy, costs, power consumption. For these reasons, positioning in challenging conditions, such as indoor

V. Pasku was with the University of Perugia - Engineering Department, via G. Duranti, 93 - 06125 Perugia Italy.

G. De Angelis is with Regione Umbria (the Regional Government of Umbria), Perugia Italy.

A. De Angelis, M. Dionigi, A. Moschitta, and P. Carbone are with the University of Perugia - Engineering Department, via G. Duranti, 93 - 06125 Perugia Italy.

D. Arumugam is with California Institute of Technology.

D. Ricketts is with the North Carolina University, Department of Electrical and Computer Engineering, Raleigh, NC, 27695 USA.
Index Terms-Magnetic field positioning, Localization systems,

environments, Non-Line of Sight (NLoS) situations, and highaccuracy applications, is gaining more and more attention in the commercial and scientific community. Thus, systems based on local references that require to triangulate the position of a mobile node with respect to a set of local beacons or reference positions, have been developed and proposed in the literature. According to [9] there are as many as 200 startups competing for visibility in the emerging indoor location and proximity marketing arena. Thus, there is no doubt that the topic of accurate outdoor and indoor positioning, localization and navigation will still gain momentum in the years to come.

While current users seem to accept technological limitations, there is a mounting need and requests to use seamless outdoor-indoor navigation services. This need is proved by technological foresight reports such as [10] where it is stated that the actual growth in active deployments, however, has been slower than expected, perhaps due to accuracy or the scope of solutions offered. Similarly there is a commercial interest in chipsets and systems capable of providing location information such as those described in [11], [12]. At the same time research centers are actively involved in this topic, with more than 4000 journal and conference articles available in IEEExplore under the search terms indoor localization. On the other side, research-funding agencies are granting funds to research groups and investigators, exploring the topic of indoor positioning and localization [13]-[16].

Thus, localization awareness is becoming an essential attribute of many commercial, public services and wireless networks. However, there is no single technology that appears to dominate the others with respect to accuracy, power consumption and portability, in all practical scenarios. In fact, proposed solutions are based on several approaches like the usage of ultrasound, optical, Ultra-Wideband (UWB), Radio Frequency (RF) narrowband signals, magnetic fields or inertial sensors. A short overview of the proposed solutions and their main characteristics will be given in Section II.

The large extent of the literature on the broad subject of positioning is testified by the fact that, during the last 10 years, several surveys have been conducted and published in the field of positioning [17]-[27]. Among all the solutions included in these surveys, the usage of magnetic fields is an interesting choice for selected applications, due to certain inherently beneficial properties. However, to the best of the authors' knowledge, none of the mentioned surveys has been focused specifically on Positioning Systems (PSs) based on low frequency magnetic fields. Therefore, an overview of magnetic field based positioning systems is of interest for the scientific community, and thus constitutes the main scope of the present paper.

In particular, a broad overview of different technologies (C2017 IEEE. Personal use of this material is permitted. Permission from IEEE must be obtained for all other uses, in any current or future media, including reprinting/republishing this material for advertising or promotional purposes, creating new collective works, for resale or redistribution to servers or lists, or reuse of any copyrighted component of this work in other works. DOI: $10.1109 /$ COMST.2017.2684087 
proposed for implementing positioning systems is first provided, to place the paper in the proper context. Subsequently, a detailed analysis regarding the usage of magnetic fields as the main mechanism to distribute information about range and, - in turn - position information, is presented. Specifically, proposed techniques and obtained results are described and compared.

The reminder of the paper is organized as follows. In Section II a broad overview of the main characteristics for various positioning technologies is given. In Section III, magnetic-field based positioning systems are analyzed in more detail, and a classification is provided. Furthermore, Section IV is devoted to systems exploiting the terrestrial magnetic field, while artificially-generated magnetic fields are analyzed extensively in Section V. Finally, a discussion and comparison of results is provided in Section VI, and conclusions are drawn in Section VII.

\section{Positioning Technologies Overview}

\section{A. Positioning methods}

From a theoretical standpoint, all position-measurement principles entail the inversion of a measurement model. Such model may require the transmission of specific signals and the measurement of received signals, relating the measured parameters to the distance between the mobile node and a beacon. In this case, Received Signal Strength (RSS), Angle of Arrival (AoA), and Time of Flight (ToF) are typically measured [19], [20], [28]. The fundamental operation of such positioning methods is depicted in Fig 1 (a)-(c).

Alternatively, the measurement model may be formulated using the so called fingerprinting approach. This approach implies the measurement of location-dependent properties at known locations. Thus, a database is built during the initial system deployment phase. Then, the properties measured during the operational phase are matched to those contained in the database. The operation of the fingerprinting method is illustrated in Fig. 1(d).

\section{B. Classification Scheme}

A broad classification of positioning systems is illustrated in Fig. 2. According to this classification scheme, the two main categories that can be formulated are Global PSs and Local PSs. Systems in the Global PSs class include the widely-used GNSSs that employ triangulation and time-of-arrival (TOA) to determine $3 \mathrm{D}$ position providing global coverage. Due to the high frequency of $1.1-1.5 \mathrm{GHz}$, these signals are delayed or severely attenuated when propagating through lossy bodies [29], which results in position errors in the order of tens of meters. In the presence of clutter, the multi-path phenomenon may also cause errors on the scale of many tens of meters depending on the measurement environment [30]-[32] .

For these reasons, when dealing with challenging environments and applications, it is necessary to implement other positioning systems, pertaining to the Local PS class. As summarized in Fig. 2, the main approaches in the Local PS class, are based on radio-frequency (RF) propagation, ultrasound, inertial navigation, optical systems and magnetic field. In the following, the main characteristics of each technology, such as cost, accuracy, scalability, power consumption, complexity are provided and compared.

\section{RF Positioning Systems}

1) Narrowband RF Technologies: Several positioning systems in this category are implemented using Wireless Area Network (WLAN) technology, typically by proximity detection or fingerprinting techniques. The main advantage of WLAN is that positioning can be considered as a complementary service, obtained without additional cost and complexity. On the other hand, the main disadvantage is the low accuracy of the order of 1-50 m, depending basically on the number of fingerprinting points [17], [20], [33], [34]. Moreover, the deployment of the access points usually is not optimized for positioning applications, but rather for communication purposes. However, positioning systems based on ad hoc WiFi networks have been developed, as is the case of WASP [35], which is based on time-of-arrival (TOA) measurements. The reported results present a localization error that varies from $0.11 \mathrm{~m}$ to $0.61 \mathrm{~m}$ across several indoor and outdoor environments with operating ranges of tens of meters.

Furthermore, other positioning systems are realized using standard existing hardware, such as ZigBee [36]-[39] or Bluetooth [40], [41] devices. They require low power consumption and show low complexity and costs. These systems are usually based on RSS measurements and the performance in indoor environments may be degraded by multipath or in-band interference. Typical accuracy of ZigBee based solutions combined with multipath mitigation techniques is of the order of 1-3 m, while Bluetooth systems offer room level accuracy. Instead, ZigBee solutions combined with precise time measurements offer accuracies of the order of tens of centimeters at the expense of a higher cost [37]. Typical operating ranges of ZigBee and Bluetooth devices in indoor environments are in the interval $20-30 \mathrm{~m}$ or 5-10 $\mathrm{m}$ respectively.

2) UWB: To improve accuracy and multipath resilience, UWB signals have been proposed. Compared to narrowband RF signals, these signals offer superior range resolution through their fine time resolution [42]. In fact, coupled with TOA or time-difference of arrival (TDOA) measurements, the UWB technology consents an accuracy of a few $\mathrm{cm}$ over several hundred meters of operating range in LoS conditions [43]. However, due to the use of higher frequencies, UWB systems are affected by NLoS conditions and cluttered environments [42]. Practical measurements of UWB systems in NLoS environments demonstrate meter-order position errors for a system range of about $20 \mathrm{~m}$ [44]. While the technological development has decreased the UWB systems costs, the main disadvantages of such solution are power consumption, which led to research on low duty-cycle solutions, and potential interference to other services, which causes power limitations for commercial UWB systems [45]-[48].

3) Cellular-based Localization: Cellular-based localization systems have been motivated primarily by the E-911 service regulations, required by the Federal Communications Commission (FCC). Existing cellular techniques include TDOA, 


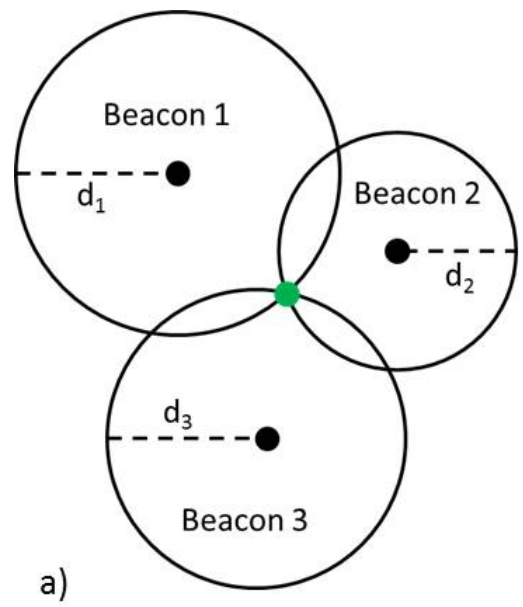

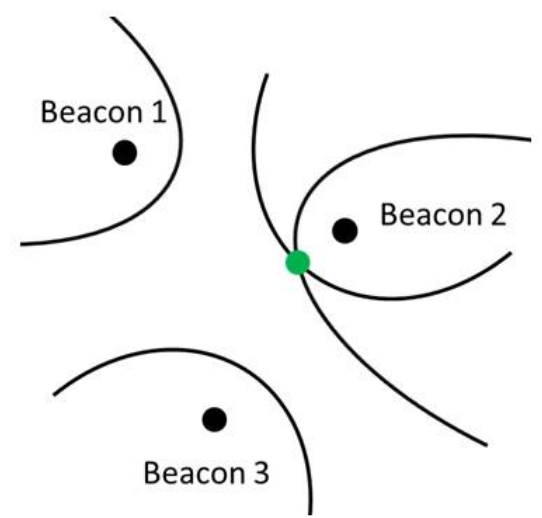

b)

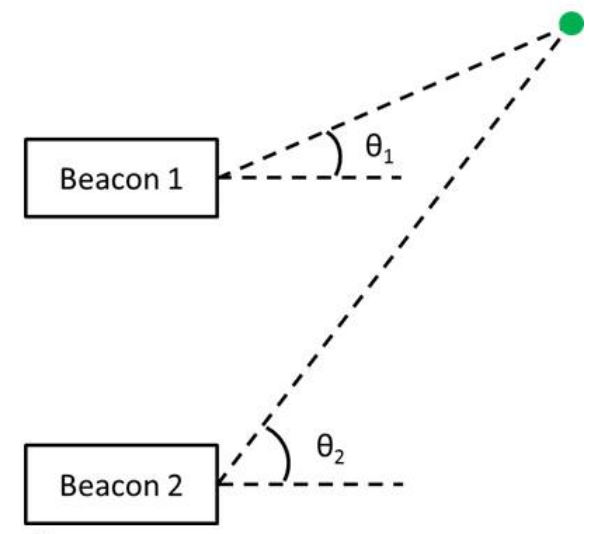

c)

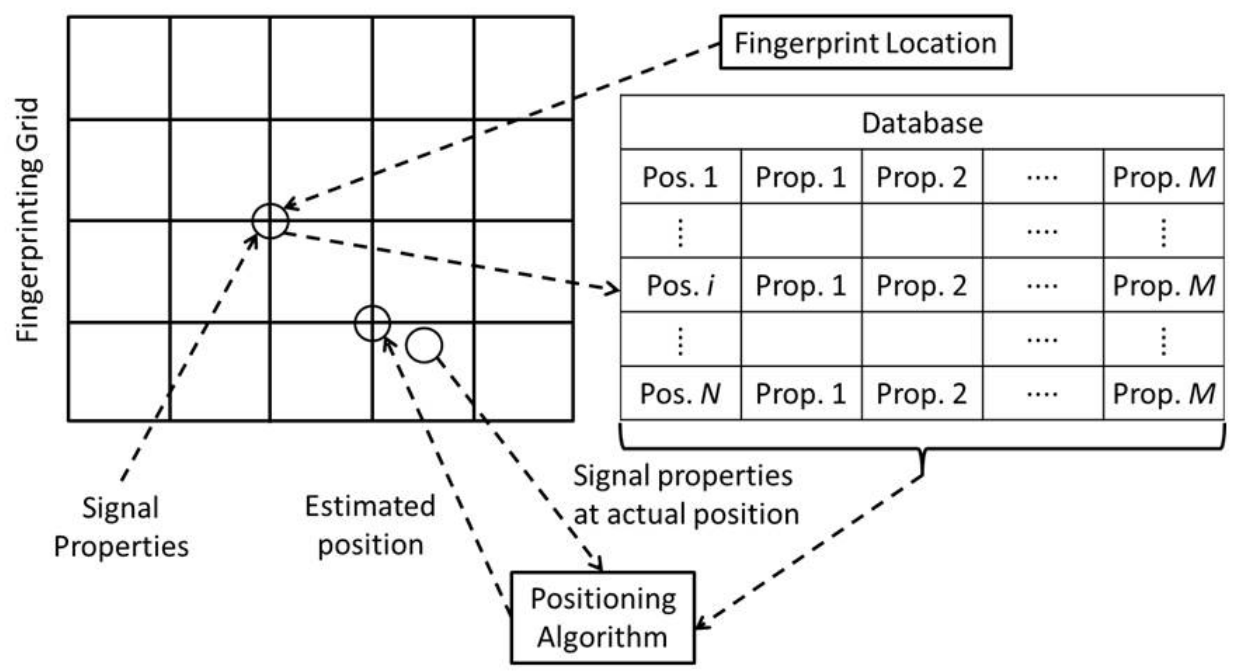

d)

Fig. 1. Schematic representation of the main positioning methods. a) Positioning obtained by a multilateration approach based on ToA or RSS measurements; b) Positioning obtained by TDoA measurements; c) AoA measurements and d) Fingerprinting approach.

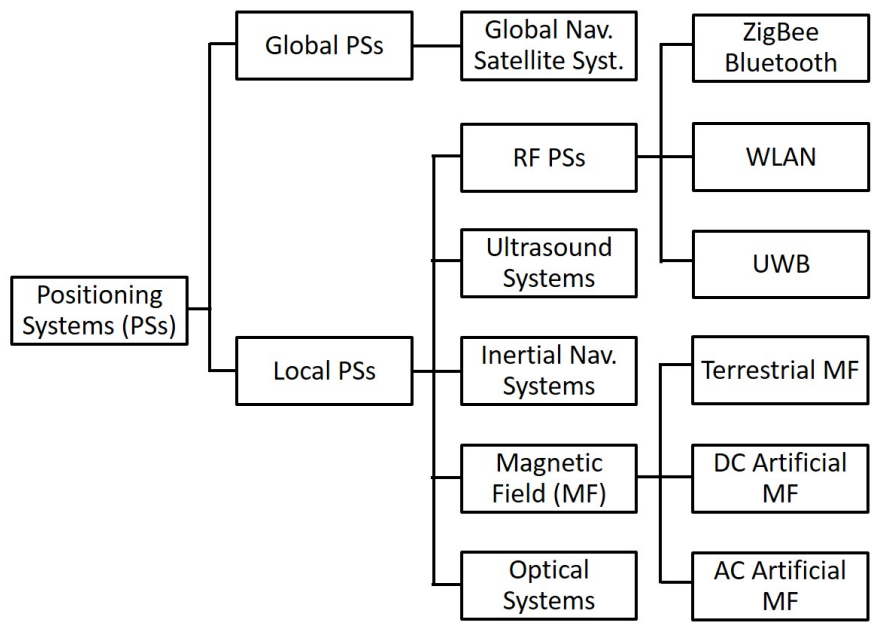

Fig. 2. Schematic representation of the main available positioning systems.

AOA or hybrids [49]. Practical measurements of systems based on the E-911 requirements demonstrate position measurement errors on the order of many tens of meters to hundreds of meters in NLoS or multi-path environments [50], [51].
4) RFID: RFID positioning systems are typically implemented as ultra-high-frequency (UHF) systems or surfaceacoustic wave (SAW) systems. UHF RFID systems generally operate by measuring the backscatter from a passive tag or by measuring the RSS of an active tag [22]. These systems typically operate in the $900 \mathrm{MHz}$ industrial, scientific and medical (ISM) band and are often strongly attenuated when propagating through lossy objects [52], resulting in range measurements with meter-order errors in practical environments [53], [54]. SAW RFID systems typically operate at $2.4 \mathrm{GHz}$ and experience similar problems to UHF RFID systems, although SAW systems also suffer heavily in multipath environments due to use of the TOA localization approach [55].

5) Other RF Technologies: AM band transmissions have been used as signals-of-opportunity in the past to determine the position of a passive receiver, with position errors on the order of tens of meters [56], [57]. In this approach, the AM broadcast signals from nearby stations are received and digitized, and the carrier phase measurements are used to estimate position.

Another approach using signals-of-opportunity is based on FM band transmissions to enable positioning [58], [59]. In this 
approach, the position information of a target is found relative to a FM co-located transmitter. Due to the low bandwidth of FM transmissions, the approach provides low range resolution with ranging error on the order of a few hundred meters to about a kilometer or greater [59].

\section{Inertial}

Inertial Navigation Systems (INSs) provide estimates of acceleration, velocity and orientation by processing data acquired from an Inertial Measurement Unit (IMU) [60]. The relative position of a user with respect to an initial position is obtained by double integration of measured acceleration. Absolute positioning information may be obtained by fusing INS with complementary sensors. The main advantage of INS is the independent operability, allowing navigation in environments where infrastructure installation is problematic. However, INSs show a performance degradation over time and space due to the double integration process [60]. To increase the time span during which INS can provide data with a limited error, foot-mounted INS implementing Zero velocity-UPdaTes (ZUPTs) may be used [61], [62]. Low cost INS have started to be common in the market and are characterized by a low computational effort. Typical accuracy of foot-mounted INS is of the order of $0.1 \%-0.4 \%$ of the traveled distance.

\section{E. Ultrasound}

Ultrasonic pressure waves are a popular class of signals, relying on a well-established background in medical scanning and nondestructive testing, mostly based on ToF measurements. The use of ultrasonic waves is a low power and low cost approach, providing an accuracy of one centimeter or better in a range of less than 6 meters [63], [64]. Due to the small size of sensors and low complexity of the acquisition section, ultrasonic systems can easily be used together with other techniques, leading to hybrid systems. Moreover, due to LoS requirements and limited range, the ultrasound positioning systems mentioned in the literature usually require the deployment of several beacons.

\section{F. Optical}

The technological advancement in the field of actuators and detectors for image applications, i.e. lasers and sensors, in parallel with the development of image processing algorithms, is the main enabler for positioning systems based on cameras or optical signals [65], [66]. These systems cover a wide field of applications with an accuracy from tens of micrometers to decimeters. Optical positioning systems can be divided in two main categories: systems where a mobile sensor is to be located, and thus reference information is required, and systems where static cameras locate moving objects in images without reference information. The systems in the first category usually rely on the detection of features in images and on matching such features with a database containing position information [65]. Such systems show accuracies of the order of decimeters and costs from moderate to high. On the other hand, the goal of camera-based positioning systems without reference information is to observe position changes directly from the acquired images. Single or multiple static cameras can be used in order to track objects with high update rate. The obtained accuracies are of the order of tens of micrometers [67], [68] and the systems show very high costs.

\section{G. Magnetic Fields}

The localization problem can be solved also by using very low frequency magnetic fields. This approach has interesting features, such as the possibility of not being easily blocked by artifacts such as indoor walls or groups of people in NLoS situations [69]. Moreover, magnetic fields are relatively easy to generate and detect, with a well-developed underlying theory. They do not suffer from the effects of multipath and fading that are typical of other technologies, such UWB. The main disadvantage is the relatively short operating range. Since coupling quickly decreases with distance, the usage of AC signals has been recently proposed, tuning high $\mathrm{Q}$ wire loop antennas to resonance in order to increase transmission efficiency. This approach, described in the following sections, requires simple hardware, and consents an accuracy of a few centimeters in a range of a few tens of meters. Magnetic positioning is already a competitive technology, as proven by fielded motion tracking solutions that are already on the market [70], [71].

\section{H. Main Characteristics Comparison}

In order to provide a clear overview of the main advantages and disadvantages, the fundamental characteristics of different classes of positioning systems are compared in Table I] In particular, accuracy, cost, power consumption, operation range, complexity, and limitations of different technologies have been considered. The comparison shows that there is no single technique that completely dominates with respect to other solutions, and thus the optimal choice depends on the specific field of application. Moreover, different techniques can be combined for an increased performance.

\section{Magnetic Field Positioning}

As discussed in the previous section, the numerous technologies proposed for positioning in GNSS-challenged environments are subject to limitations and are affected by environment configuration issues. The use of quasistatic magnetic fields for positioning purposes successfully addresses some of these fundamental issues. Specifically, positioning systems based on magnetic fields are not prone to multipath degradation, provide excellent penetration properties through most non-metallic materials, and can operate in obstructedline-of-sight conditions. Additionally, the use of magnetic fields typically results in a lower architectural complexity with respect to radio-frequency and microwave technologies.

Exploiting such advantages with respect to competing technologies, several magnetic positioning systems (MPSs) have been recently proposed in the literature [20]. In particular, a division in three main groups can be observed. Specifically, in the first group, positioning is achieved by using the Earth's 
TABLE I

TECHNOLOgIES MAIN CHARACTERISTICS COMPARISON

\begin{tabular}{|c|c|c|c|c|c|c|}
\hline Technology & Accuracy & Cost & Power Consumption & Operation Range & Complexity & Limitations \\
\hline UWB, Section II-C2 & Centimeter level & medium to high & medium to high & Hundreds of meters & medium to high & NLoS operation \\
\hline WiFi, Section II-Cl & $2-50 m$ & low & medium & Hundreds of meters & low to medium & Low accuracy \\
\hline Bluetooth/ZigBee, Section/II-C1 & $1 \mathrm{~m}-$ room level & low & low & 10 to 30 meters & low & Multipath \\
\hline Inertial, Section III-D & $0.1 \%-0.44 \%$ & low to medium & low & Limited by the error & low to medium & Limited time and space \\
\hline Ultrasound, Section III-E & Centimeter level & low & low & 5 to 6 meters & low to medium & Require LoS \\
\hline Optical, Section $[\mathrm{II}-\mathrm{F}]$ & Submillimeters to decimeters & medium to very high & low to medium & Scalable from $\mathrm{m}$ to $\mathrm{km}$ order & medium to very high & Require LoS \\
\hline Cellular-based, Section II-C3 & Hundreds of meters & low to medium & low & order of kilometers & low to medium & Low accuracy \\
\hline Magnetic Fields, Section II-G & Subcentimeter to 1 metter & low to medium & medium to high & $10 \mathrm{~m}$ to $100 \mathrm{~m}$ & low to medium & Short range \\
\hline Other, Section II-C5 & Tens of meters to a kilometer & low & low & order of kilometers & low to medium & Low accuracy \\
\hline
\end{tabular}

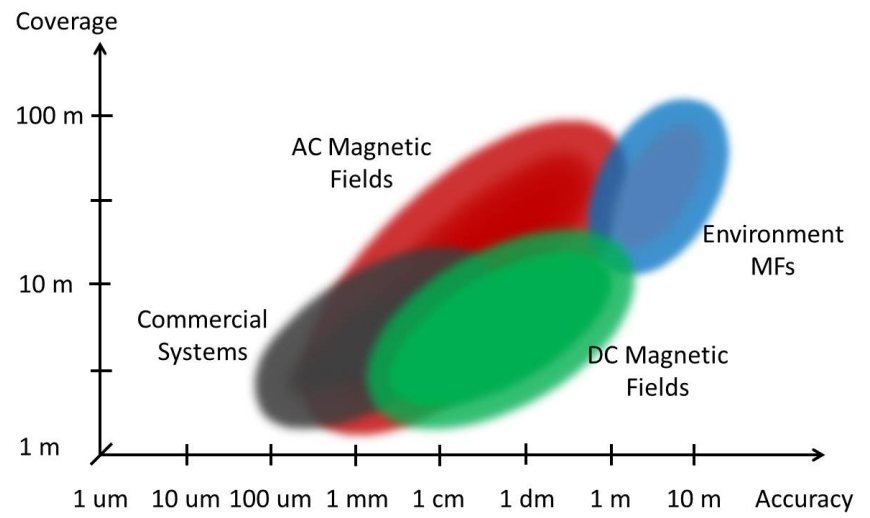

Fig. 3. Coverage-Accuracy characteristics of magnetic field-based positioning systems.

magnetic field alone [72], [73] or local anomalies of the ambient magnetic field [74]. In the second group, generated DC magnetic fields [75] are employed, while the third group is based on generated AC magnetic fields [76], [69]. Moreover, a fourth group is represented by application-specific commercial systems. All these techniques will be reviewed in the following part of the paper. Each group is characterized by different properties. In particular, accuracy and coverage are directly related to the underlying technology. A schematic representation of the accuracy-coverage characteristics of the above main groups is shown in Fig. 3. The best coverage is offered by systems based on ambient magnetic fields, while the best accuracy is usually offered by application-specific commercial systems. Instead, AC and DC magnetic field based PS represent a wide set of choices. Usually, the location of the PS in the considered representation in Fig. 3 is determined by the cost and complexity. In particular, higher system cost and complexity lead to higher coverage and/or better accuracy.

Examples of systems based on quasistatic magnetic fields also include direction finding (DF) systems, such as avalanche rescue transceivers (also known as ARVA), magnetic-coupling based sensing, and near-field phase difference techniques. In most applications, the use of quasistatic fields is motivated by the need to operate in or around lossy dielectrics such as ground, water, or snow cover during an avalanche.

\section{Terrestrial Magnetic Field}

One of the magnetic positioning strategies proposed in the literature exploits the Earth's magnetic field. This class of systems are usually obtained by fingerprinting techniques and show high coverage area. In particular, the local anomalies and disturbances are typically used for mapping the magnetic fingerprint of the environment in which the positioning system is to be deployed, as demonstrated in [74]. A schematic representation is shown in Fig. 4. A grid of known surveyed positions is first defined in the environment. Using an electronic device equipped with magnetic field sensors and a processing unit, for each grid position a database entry is generated. Then, in the operating phase, the actual measurement is compared against the database and a position estimate is obtained. The main limitation of this type of systems is the requirement of a cumbersome initial mapping process. Moreover, the obtained system accuracy is directly related to the grid size.

Measurements of the Earth's magnetic field were used for a pedestrian navigation system in [77]. In particular, the magnetic field information was used for aiding an INS in order to mitigate gyroscope errors. Tracking results in urban canyon scenarios showed that fusion with magneticfield information, even with local disturbances, allows for a considerable error reduction with respect to traditional inertial integration techniques.

Similarly, the ambient magnetic field anomalies generated by environment characteristics were used to build magnetic maps for indoor localization in [78]. In particular, different mobile phones equipped with magnetometers were used and room-level positioning accuracy was obtained.

The disturbances of the Earth's magnetic field, caused by structural steel elements in buildings, have been used in [79] in order to measure users location. Magnetic fields are measured by using an array of HMC6343 e-compasses and the measurements are compared against a previously obtained magnetic map. The database is built in a $0.6 \mathrm{~m}$ grid in the considered environments. The obtained performance across multiple floors of two different buildings is characterized by a positioning error of 2-6 $\mathrm{m}$, depending on the considered environment. By applying different constraints to the searching algorithm, the improved accuracy is within $1 \mathrm{~m} 88 \%$ of the time.

MaLoc (Magnetic fingerprinting based indoor Localization) is another indoor positioning system which is based on ambient magnetic field measurements using smartphones [80]. The MaLoc system consists of a client running on smartphones and a server, where a novel reliability-augmented particle filter is implemented. The client collects magnetic and inertial sensor data and performs step counting, heading change between two contiguous steps, and accesses magnetic values in each step. The preprocessed data are sent to the server, which performs 
computations and sends updated locations to each user. MaLoc does not impose any restriction on smartphone's orientation and, due to the hybrid magnetic measurement model, does not require previous calibration. The system has shown an average accuracy of the order of 1-2 $\mathrm{m}$ in a large building.

Geomagnetic fields and radio ranging signals have been used as information sources in order to build landmarks in a SLAM and relocation framework which employs the RaoBlackwellized particle filter and grid-based landmark construction in [81]. The system was tested in three different test environments, where a robot was driven along given paths performing magnetic SLAM. Then, the robot was resituated at different predefined relocation points. Using the fingerprinting results estimated during the SLAM, the relocation performance has shown an accuracy better than $20 \mathrm{~cm}$ in all the cases.

Magicol [82] is another magnetic field based indoor localization and tracking system that makes use of the geomagnetic field anomalies. Magicol leverages the locally disturbed magnetic signals as location-specific signatures that are acquired by magnetometers, which are commonly found on smartphones. Similarly to MaLoc, Magicol is composed by a mobile client, which performs data logging and motion state detection, and a cloud service, which consists of two subsystems: a location database construction and a location inference engine. The location inference engine matches the current magnetic signals with a magnetic map by using an augmented particle filter. Magicol has been evaluated in three representative indoor environments: an office floor, an underground parking lot, and a supermarket. The operating areas are $4000 \mathrm{~m}^{2}, 3850 \mathrm{~m}^{2}$ and $1900 \mathrm{~m}^{2}$, respectively. When using the magnetic field alone the performance was characterized by 90th-percentile localization accuracy of $5 \mathrm{~m}, 1 \mathrm{~m}$ and $8 \mathrm{~m}$, respectively. Instead, the fusion with WiFi signals has shown 90th-percentile accuracy $3.5 \mathrm{~m}$ for localization and $0.9 \mathrm{~m}$ for tracking in the office environment.

Another system based on magnetic field disturbances as the primary source of information is described in [83]. In particular, using only the information of the magnetometer and the accelerometer of a smartphone as an input for a particle filter, good localization capabilities have been obtained. Localization performance was characterized by a position error better than $1.2 \mathrm{~m}$ and an orientation error better than $40^{\circ}$ in the case of a circle trajectory. In the case of a straight line trajectory, better results have been obtained.

\section{Artificially Generated Magnetic Fields}

The magnetic field-based positioning systems described in the previous section were developed by considering the Earth's magnetic field and its local disturbances, introduced by the characteristics of the environment. Conversely, the magnetic field-based positioning systems described in the following part of the paper are based on fields that are artificially generated by coils.

Due to the direct relation between position and generated magnetic field, the position of a user with respect to field sources can be inferred using magnetic field measurements. In this way, systems for self positioning, navigation, or tracking can be realized.

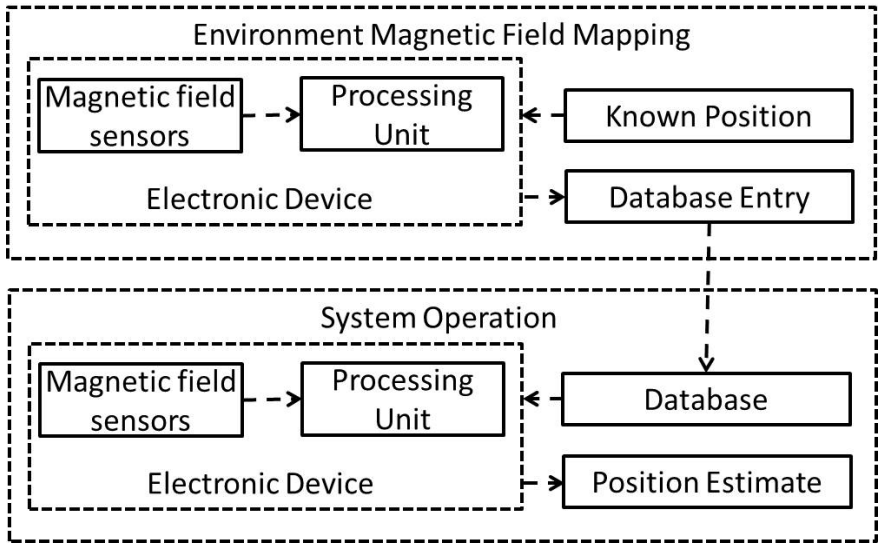

Fig. 4. Schematic representation of environment magnetic field-based positioning systems.

Depending on the operating frequency, this class of positioning systems can be further divided in DC field-based and time varying field-based positioning systems. In the first case, the field source could be realized also by using permanent magnets instead of coils.

Artificially-generated magnetic-field systems are generally based on the magnetic dipole field expression given by [84], [85]:

$$
\mathbf{B}(x, y, z, t)=\frac{\mu_{0}}{4 \pi}\left[\frac{3(\mathbf{m} \cdot \mathbf{r}) \mathbf{r}-\mathbf{m} r^{2}}{r^{5}}\right] e^{-j \omega t},
$$

where $\mu_{0}$ is the free space magnetic permeability, $\mathbf{r}$ is the position vector from the origin to the observation point, with $|\mathbf{r}|=r, \mathbf{m}$ is the coil's magnetic moment, $\omega$ is the operating frequency and $t$ is time. The magnetic moment is orthogonal to the coil surface and has a module $m=N I S$, where $N$ is the number of turns in the coil, $I$ is the feeding current and $S$ is the coil's surface. A schematic representation of the magnetic field generated by a coil centered at the coordinate's system origin is shown in Fig. 5. Considering a second coil inside the generated magnetic field, from Faraday's law of induction, a voltage will be induced when using time varying signals. The induced voltage at the sensor coil is given by:

$$
V(x, y, z, t)=-j \omega N_{r x} a \mathbf{B}(x, y, z, t) \cdot \hat{\mathbf{n}},
$$

where $\hat{\mathbf{n}}$ is the unit vector normal to the sensor coil, $a$ is the surface area of the sensor coil, and $N_{r x}$ is the number of turns in the sensor coil. By using $k$ fixed field generating coils with a priori known positions and orientations and a sensor coil, the unknown position and orientation of the sensor coil can be found. Alternatively, with $k$ known-position sensor coils and a generating coil, the unknown position of the generating coil can be found. The position estimation may be performed using a least-squares approach by minimizing a cost function related to the difference between the measured voltages and the theoretical position-dependent voltages. As an example, the cost function can be given by:

$$
\Phi=\sum_{i=1}^{k}\left[V_{i}^{T}-V_{i}^{M}\right]^{2}=\sum_{i=1}^{k} \varepsilon_{i}^{2} .
$$




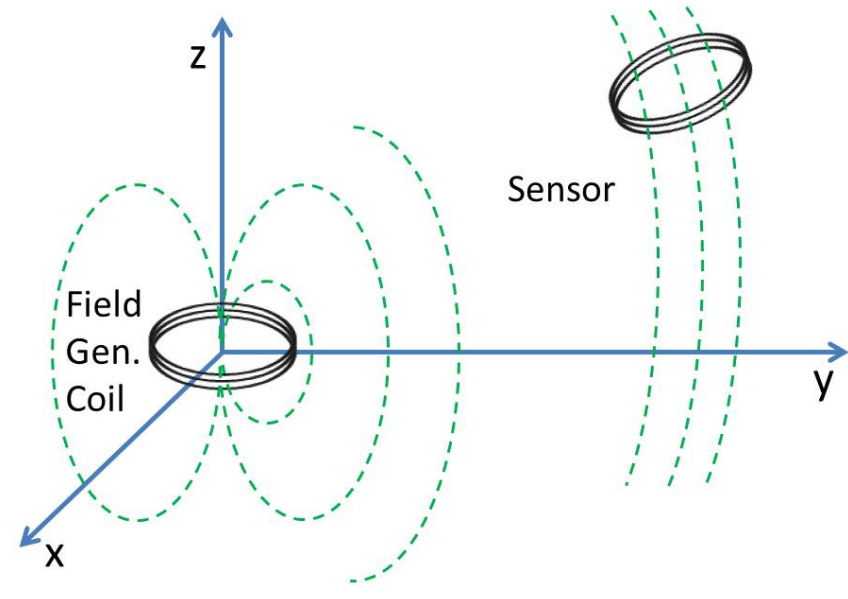

Fig. 5. Schematic representation of the basic working principle of artificially generated magnetic fields positioning systems.

Here, $V^{T}$ is the generated voltage given by (2), $V^{M}$ is the measured voltage in the sensor coil, and $\varepsilon_{i}$ is the residual error for each sensor-emitter couple measured. The estimated location is chosen as the one that minimizes the sum of the squared residual errors in (3). Similar considerations hold when using DC signals. In this case the measurements are magnetic field amplitudes instead of voltages and the cost function is built by using this magnetic field amplitude measurements instead of voltage measurements.

Time varying field based systems usually show a higher operating range with respect to DC field-based systems, as can be concluded by (2), where the induced voltage is directly proportional to the operating frequency. Moreover, instead of simple coils, often resonant coils are used. The resonance properties improve the operating range and/or decrease the required system current consumption.

The magnetic dipole model in (1) and (2) may be refined by employing more accurate expressions. As an example, the model in [86], [87] may be used to calculate the mutual inductance between arbitrarily-oriented coils. Such advanced models allow for improving position estimation accuracy, especially when orientation information is needed in addition to position information, e.g. for six-degrees-of-freedom localization applications. However, this comes at the cost of increased computational complexity.

\section{A. DC Magnetic Field-Based Systems}

Static (i.e. constant over time) magnetic fields are used for positioning purposes and both low and high operating range systems are realized. In the remainder of this section, systems with an operating range lower than $2 \mathrm{~m}$ are classified as low operating range, while systems with an operating range higher than $2 \mathrm{~m}$ are classified as high operating range.

1) Low operating range: The usage of DC magnetic fields in short range tracking applications has been described in [88], [89]. In particular, a small cylindrical shaped permanent magnet of diameter $0.5 \mathrm{~cm}$ and length $1.2 \mathrm{~cm}$ has been tracked over an area of $0.5 \mathrm{~m} \times 0.5 \mathrm{~m} \times 0.5 \mathrm{~m}$ by using an array of 64 three-axis magnetic sensors. A mean accuracy of $3.7 \mathrm{~mm}$ has been obtained for real time tracking of three magnets inside the area delimited by the sensors array, simultaneously. Potential applications include medical systems such as the tracking of an endoscope. Moreover, in [90], a global trunk orientation and relative hand pose estimation method is presented. A permanent magnet combined with 3D magnetometers and 3D inertial sensors are used and their information is fused through an Extended Kalman Filter. The system performance has been evaluated through multiple experiments in which the worst mean position error was approximately $2 \mathrm{~cm}$. Moreover, hand trunk and global trunk orientation errors of approximately $2.3^{\circ}$ and $8.6^{\circ}$ were obtained, respectively. The above performance was obtained for simultaneous movements of both hand and trunk.

2) High operating range: High range positioning systems based on DC magnetic fiels are usually characterized by a high current and power consumption. In [75], [91] the use of DC artificially-generated magnetic fields was investigated. In particular, the magnetic field generated by $50 \mathrm{~cm}$ diameter and 140 turns coils, fed by a current of $12 \mathrm{~A}$, was acquired by Honeywell magnetoresistive transducers. Results showed that a mobile user can perform self-localization exclusively based on the measurement results from the tri-axis magnetometer. The observed accuracy was of the order of $10 \mathrm{~cm}$ in the case of 2D positioning over an area of $15 \mathrm{~m} \times 15 \mathrm{~m}$, and approximately $50 \mathrm{~cm}$ in the 3D case. The localization based on measurements by the magnetometer of a smartphone have been also obtained, albeit with lower accuracy. In particular, planar positioning with an accuracy of $0.8 \mathrm{~m}$ has been obtained over an area of $7 \mathrm{~m} \times 7 \mathrm{~m}$, or approximately $1.5 \mathrm{~m}-2 \mathrm{~m}$ in the 3D case over a volume of $4 \mathrm{~m} \times 4 \mathrm{~m} \times 3 \mathrm{~m}$. In order to improve the positioning performance, the developed system has been integrated with an IMU-based positioning system [92], [93]. An improvement of the update rate of the magnetic positioning system and the tracking performance of the IMU-based system has been obtained. Finally, a decentralized version of the previous prototype has been developed and described in [94].

Moreover, in [95], distributed beacons were used to generate magnetic fields with a code division multiple access approach, yielding centimeter-level accuracy in harsh non-line-of-sight conditions. Specifically, DC magnetic fields were modulated by $630 \mathrm{~Hz}$ pseudo-random sequences to distinguish beacons by code-division multiple access. The low-frequency operation, however, entails high current consumption, and poses limitations on the maximum range (approximately $10 \mathrm{~m}$ ). Therefore, a relatively large number of transmitters must be deployed for a usable operational range.

\section{B. Time Varying Magnetic Field-Based Systems}

Similarly to the case of DC magnetic fields, also AC magnetic fields are used in positioning applications. However, this class of systems is usually characterized by lower current consumption and/or higher operating ranges. Thus, in the remainder of this section, a range of about 3-4 m is used in order to distinguish between low and high operating range. Moreover, in this case, also a distinction between above or underground operation is considered. 
1) Low operating range: Quasistatic positioning systems designed for above ground short-range operation have previously focused in the ultra-low-frequency region. In this approach, the transmitter to be located emits a tone at a frequency of less than about $4 \mathrm{kHz}$ which is sensed using receivers and inverted for the position of the transmitter [96]-[104]. Due to Faraday's law, the low-frequencies used here do not provide sufficient signal-to-noise ratios for operation beyond about 1$1.5 \mathrm{~m}$ range. However, due to the long-wavelengths and from use of quasi-static magnetic fields, operation within this short range is often unimpeded by LoS blockage, therefore allowing use in short-range NLoS motion-tracking applications [105][110].

In [111] a method of localization of a three-axis sensor coil is described. In particular, the method is based on several mathematical equations rather than matrix computation and nonlinear optimization, to directly calculate the receiver position with respect to the transmitter. A mean positioning error of $1 \mathrm{~mm}$ and a mean orientation error of $1^{\circ}$ are obtained over a test volume of $0.5 \mathrm{~m} \times 0.4 \mathrm{~m} \times 0.4 \mathrm{~m}$ covered by thre different three-axis transmitters. The described method has been extended to the case of tracking a three-axis transmitter by using a single axis rotation coil [112].

A magnetic position tracking system for radiation therapy is described in [113]. It uses four single axes transmitting coils and an implantable transponder provided by a bi-axial magnetoresistive sensor. The magnetic field sensed by the transponder implanted in the tumor is transmitted through wireless or wired interface to a computer where the position is estimated. The system shows an update rate of approximately $33.3 \mathrm{~Hz}$ and an error of less than $2 \mathrm{~mm}$. Similarly, an ambulatory position and orientation tracking system [114] and a wearable activity recognition system [115] based on magnetic fields generated by coils have been developed and described. Results have shown an accuracy suitable for the application requirements in both the cases.

In [116] a 2D positioning system for short to long ranges based on magneto-quasistatic field and Complex Image Theory (CIT) was described. The technique enables accurate $2 \mathrm{D}$ position and orientation sensing using magnetoquasistatic fields, with only a single anchor, or a single reference node, and does not rely on tri-angulation/lateration.

Ranging of a mobile passive, battery-less, resonant loop was demonstrated in [117]. Here, it was shown that the use of magnetoquasistatic fields and loops in coupled magnetic resonances with each other enables passive short range position and orientation measurements. An average range error of 0.61 $\mathrm{cm}$ was obtained for operating ranges up to $1.7 \mathrm{~m}$.

2) High operating range: Quasistatic positioning systems designed for above ground long-range operation have previously focused in the medium-wave region. One example is the use of magnetoquasistatic fields using a frequency of about $2 \mathrm{MHz}$ [118]. In this approach, the transmitter to be located emits a tone at a frequency of $2 \mathrm{MHz}$ which is sensed using receivers located within the quasistatic region of the source. The corresponding wavelength of the emitted field was about $150 \mathrm{~m}$ compared to the system operation range of about up to $30 \mathrm{~m}$. Within this region for above ground free-space operation, the fields of the transmitter are primarily magnetic in nature such that weakly conducting dielectric bodies do not perturb the fields considerably. Measurements using this system have been conducted and fitted to an arbitrary powerlaw best fit curve to derive standard deviation errors on the order of about a meter [118]. Due to use of an arbitrary power law relationship to match theory to measurements, the approach must rely on RF-fingerprinting techniques to map the measured power in the area prior to solving for location based on the map [118].

Another example of quasistatic positioning is the use of near field phase difference between the electric and magnetic fields of a quasistatic source [119]-[121]. Within the near field of an electromagnetic source, the electric and magnetic fields are out-of-phase. Very close to the source, the fields are $90^{\circ}$ out of phase, and they gradually become in-phase as they approach the edge of the near field region. Measurements of the phase difference therefore can provide an estimate of distance from the source within the quasistatic region. A frequency of about 1.2-1.3 MHz has been used to demonstrate distance sensing accuracy of approximately $0.3-1 \mathrm{~m}$ over distances up to about $50 \mathrm{~m}$ [119]. Because the phase change is negligible very close to the source (normalized to wavelength), near field phase difference techniques must generally operate close to the induction region to benefit from the rapid phase change, which often requires use of frequencies in the medium-wave region as opposed to lower frequencies. In the presence of dielectric obstacles, which have a strong electric response, it is expected that the electric field component to be perturbed, resulting in a position error. Because the phase difference does not change as a function of source orientation, the technique cannot provide orientation sensing.

In the ultra-low frequency range, the magnetic selfpositioning system described in $[122]-[124]$ provides both $2 \mathrm{D}$ and $3 \mathrm{D}$ positioning with sub-meter accuracy. In particular, a mean accuracy of $0.8 \mathrm{~m}$ and $0.4 \mathrm{~m}$ have been obtained for the 3-D and 2-D localization scenario respectively with an evaluated maximum operating range up to $100 \mathrm{~m}$. This system is based on the usage of 2 or 3 square coils with side length of $1 \mathrm{~m}$ operating at slightly different frequencies in the interval between $500 \mathrm{~Hz}$ and $800 \mathrm{~Hz}$. The involved transmitters require a current consumption of 7-8 $\mathrm{A}$ in [124] and $0.1 \mathrm{~A}$ in [122], [123]. The receiver is based on a 3-axis magnetometer and a 24 bit Analog to Digital Converter (ADC). The acquired signal is then processed by a nine channel non-coherent detector based on software lock-in amplifiers and the result is used as the input of the localization algorithm based on simulated annealing. A further developement is described in [125]. In particular, a receiver based on a tablet or a smartphone is located in an array of distributed beacons in the environment operating at different frequencies below $20 \mathrm{~Hz}$. Square beacons with side length of $50 \mathrm{~cm}$ fed by a current of $10 \mathrm{~A}$ have been used, with a power consumption of about $160 \mathrm{~W}$. The localization method is based on a proximity algorithm. Successful system operation has been proven by in field tests and several simulations.

Pirkl and Lucowicz in [126] have described the design, implementation and evaluation of a magnetic positioning system 
based on magnetic resonant coupling. The system was built using 3-axis transmitter coils with dimensions $16 \mathrm{~cm} \times 16 \mathrm{~cm}$ $\times 16 \mathrm{~cm}$ and 3 -axis sensor coils with dimensions $2 \mathrm{~cm} \times$ $2 \mathrm{~cm} \times 2 \mathrm{~cm}$. The system architecture was based on a TDMA approach with RF syncronization and an update rate of $30 \mathrm{~Hz}$. The transmitters were characterized by a maximum operating range of approximately $4 \mathrm{~m}$, by an operating frequency in the range [18.5 - 22.2] $\mathrm{kHz}$, peak-to-peak voltage of $16 \mathrm{~V}$ and maximum current of $170 \mathrm{~mA}$. After a calibration phase that consists in magnetic field measurements in a volume centered at the transmitter, distance information is obtained. The localization performance is shown to be dependent on the number of transmitters. In particular, a mean error of approximately $4 \mathrm{~cm}$ and $40 \mathrm{~cm}$ is obtained when respectively 4 transmitters and one transmitter are used. Moreover, a location classification procedure has been described, which has shown a success rate varying between approximately $80 \%$ and 97 $\%$ in different indoor environments such as an office floor, a large apartment and a small apartment with areas respectively $230 \mathrm{~m}^{2}, 100 \mathrm{~m}^{2}$ and $68 \mathrm{~m}^{2}$. The number of the deployed transmitters was between 6 and 8 , and each room contained at least one transmitter.

MagLoc, a 3D positioning system based on magnetoinductive fields and inertial sensors is described in [127]. Transmitters equipped with three mutually orthogonal coils wrapped on a wooden $30 \mathrm{~cm}$ cube former are deployed. Each coil consists on approximately 80 turns and a series capacitor, leading to a resonant frequency of approximately $2.5 \mathrm{kHz}$. Each coil transmits a BPSK modulated signal which is amplified by a $10 \mathrm{~W}$ audio amplifier. A supply current of $100 \mathrm{~mW}$ is required. The receiver contains three orthogonal ferrite-core coils of length $1.8 \mathrm{~cm}$. After being amplified by a low noise amplifier, the signals are digitized by a 16 bit ADC. Using an optimization process over the entire signal chain, a positioning accuracy below $0.8 \mathrm{~m}$ has been obtained in different environments, even in heavily distorted areas. Moreover, a range error lower than $50 \mathrm{~cm}$ has been obtained in an office environment for up to $70 \%$ of the measurements.

In many outdoor scenarios, where the magnetic field based positioning system nodes are placed above the Earth's surface, the magnetic field is distorted by the finite conductivity of the Earth itself. This distortion is particularly significant when high operating ranges are required. It can be taken into consiteration by using the CIT as in [128] where a demonstration of the use of CIT to correct ground eddy currents was described. An operational range up to $40.3 \mathrm{~m}$ with an average error of $11.7 \mathrm{~cm}$ was obtained. Following, in [69], magneto-quasistatic fields and CIT were employed in a system for estimating the three-dimensional position and orientation of a mobile transmitter. The system was developed with the aim of tracking an American football on the field when obstructions due to multiple players are present and consisted in a transmitter and seven receivers. The transmitter was composed of a coil with 50 turns of diameter equal to $16.5 \mathrm{~cm}$ and a class E oscillator with a total power of $0.56 \mathrm{~W}$ at $360 \mathrm{kHz}$, fed by a $9 \mathrm{~V}$ battery. A set of seven synchronized receivers was used for the signal acquisition. The analog signal of each receiving coil, after amplification, was digitized using a 16 bit ADC operating at
$10 \mathrm{MSa} / \mathrm{s}$. Experimental results showed a mean positioning error of $77 \mathrm{~cm}$ over an operating area of approximately 27.5 $\mathrm{m} \times 27.5 \mathrm{~m}$. Moreover, average inclination and azimuthal orientation errors of approximately $9.6^{\circ}$ and $2.8^{\circ}$, respectively, were observed.

In [76], [129]-[131] the design approach and performance of a planar positioning system based on low frequency magnetic fields is described. In particular, the system is based on a set of known-position resonant coils operating in frequency separation mode and a mobile receiver to be located. Each transmitter is composed by a circular coil of 20 turns with diameter of approximately $14 \mathrm{~cm}$, a parallel capacitor and a signal generator. The circular symmetry property resulting for the coplanar coil configuration is exploited to introduce a low complexity measurement model and calibration procedure. The developed system does not require any type of time synchronization between the nodes and can potentially accommodate an infinite number of users without additional infrastructure. The realized prototype, composed by commercial off-the shelf components, shows a low current consumption of the order of $20 \mathrm{~mA}$ for each transmitting node, thanks to the resonance properties. The receiver node, in addition to the resonant coil identical to that of the transmitting nodes, is composed by an instrumentation amplifier and a 12 bit ADC. The digitized signal is used as the input of the localization algorithm. Such algorithm first performs transmitter discrimination and amplitude estimation, based on the discrete-Fouriertransform. Then, it performs distance estimation and finally, by using multilateration, the location of the receiver is obtained. Experimental results in an indoor area of approximately 12 $\mathrm{m} \times 15 \mathrm{~m}$ with harsh NLoS conditions and multiple metallic structures such as drawers, cabinets, and door frames, have shown a mean accuracy of $0.3 \mathrm{~m}$. Moreover, a similar accuracy has been obtained in an outdoor area of approximately $14 \mathrm{~m}$ $\times 30 \mathrm{~m}$.

3) Underground or underwater operation: Direction finding systems operating using quasi-static fields provide ability to determine the direction of the electromagnetic source relative to the receiver locations [132]-[136]. Quasistatic direction finding systems measure the magnetic coupling between the emitter and receivers to determine null coupling directions by analyzing the vertical and horizontal components of the measured magnetic fields. Because most lossy dielectrics have a low magnetic response, quasistatic magnetic fields are the preferred choice of fields in DF systems. Past measurements using DF systems for below ground direction finding have demonstrated surface-2D location errors on the order of submeter to a few meters for detecting an emitter located below ground using receivers located above ground [132].

One class of DF systems, called Avalanche transceivers or beacons (ARVA) that operate at $475 \mathrm{kHz}$, has found practical use for the purpose of finding people or equipment buried under snow [137]-[139]. In this system, the transmitter is placed on the person or object to be found, and the receiver is held and moved progressively towards the transmitter to identify the location of the transmitter. Although the ARVA receiver is progressively moved closer to the transmitter, practical measurements demonstrate position determination errors 
at the meter-level [140]-[142].

Position location has also been demonstrated for underwater operation using magnetically coupled quasistatic fields [143]. In these applications, the transmitter to be sensed emits a tone at a frequency of below $100 \mathrm{~Hz}$, which is detected by the receivers and solved for location. Position location is determined by solving for the magnetic field vector and field gradient and through inversion for range from the sensor. Initial predicted accuracies are on the order of centimeter to meter-level accuracy in position determination, though this assumes an ideal environment [143].

\section{Commercial Systems}

Several commercial systems that exploit magnetic fields for positioning purposes, are available. They have varying target applications and requirements. As an example, the cabled motion tracking system by Polhemus [70] provides real-time position and orientation information with a sub-millimeter accuracy and an operational range of approximately $3 \mathrm{~m}$. It includes a System Electronics Unit (SEU), a power supply, one transmitter and up to four receivers. The SEU contains all the hardware and software necessary to generate and sense the magnetic fields, compute position and orientation, and interface with the host computer. The position and orientation of the sensor with respect to the transmitter frame shows a static accuracy better than $0.8 \mathrm{~mm}$ for the $\mathrm{x}, \mathrm{y}$ and $\mathrm{z}$ coordinate and $0.15^{\circ}$ for the orientation.

On the other hand, the near field electromagnetic ranging (NFER) solution by Q-Track [144] provides position information of tags with a maximum range up to $25 \mathrm{~m}$ and an RMS accuracy of $40 \mathrm{~cm}$. It is targeted at industrial and military applications. This system is based on measuring the relative phase between the magnetic and electric field in near-field propagation conditions [121], aided by pre-recorded information about the map of the specific building's radio environment [148].

The electromagnetic tracking system developed by Ascension [145] has the capability of tracking up to four different sensors with 6DOF. The system is characterized by a fast tracking with a default update rate of $80 \mathrm{~Hz}$ and an RMS position and orientation accuracy of $1.4 \mathrm{~mm}$ and $0.5^{\circ}$ respectively.

Moreover, Sixense [146] provides 6DOF wireless motion tracking systems for video games and virtual reality. They enable players to interact with games by tracking full position and orientation at all times, up to entire living room. An accuracy of the order of $1 \mathrm{~mm}$ and $1^{\circ}$ has been reported.

Finally, IndoorAtlas [147] is commercial positioning system based on the Earth's magnetic field. In particular, it is a cloudbased platform and does not require installation and maintenance of a hardware infrastructure but requires a preliminary mapping of the environment. An accuracy of the order of 1-2 $\mathrm{m}$ has been obtained by using magnetometers embedded in smartphones.

\section{DISCUSSION}

The above survey of positioning systems shows that active and passive propagation based systems operate best in LoS applications. Conversely, in NLoS applications, propagation based systems suffer reduced performance, which in some cases could yield errors of the order of several meters or greater. Often, such errors cannot be tolerated for NLoS tracking systems operating over ranges of tens of meters.

On the other hand, magneto-quasistatic systems provide considerable immunity to LoS blockage and have the ability to provide orientation sensing without having to rely on additional sensors. By using artificially generated low-frequency magnetic fields, which are only slightly perturbed by the presence of weakly conducting obstacles, such as humans and office environments, 1D- to 6D- positioning and orientation systems can be realized. However these have been previously limited to short range tracking, progressive direction finding applications, or medium-wave techniques that require RFfingerprinting or do not provide complete immunity to dielectric obstacles (use of electric fields). Recently, several magnetic field based positioning systems have been developed, with different architecture solutions and system characteristics. An overview of such systems was provided in this paper and a comparison of the main characteristics of some of them is reported in Table $\Pi$.

The high number of available indoor and NLoS positioning systems and the diversity of applications suggest that there is not a system that dominates with respect to others. Only the definition of the application requirements, such as accuracy, cost, operating range, power consumption and complexity can restrict the available options and guide the user choices. As an example, a system providing millimeter-order accuracy but only over a couple of meters range cannot be considered better than a system providing sub-meter accuracy over tens of meters range when the application at hand is pedestrian localization in office environments.

Open research issues include the miniaturization of magnetic field transmitters and receivers, power consumption reduction, and efficient system design, without affecting operating range and system accuracy. To this purpose the usage of resonance properties has shown a beneficial effect and the indoor operating range of magnetic field based positioning systems has started to become comparable to the useful operating range of RF propagation based systems.

\section{CONCLUSION}

In this paper, a survey of positioning systems based on magnetic field was provided. First, the current indoor and NLoS positioning scenario was described. In particular, an overview of the current available technologies and a comparison of their characteristics were provided. After that, the paper focused on positioning systems based on magnetic fields, which provide higher robustness in indoor and NLoS scenarios with respect to other available approaches, including ultrasound and narrowband RF solutions. Two different approaches have been used, PS based on ambient magnetic field and PS based on artificially generated magnetic field. The former approach is characterized by lower accuracy and system complexity, but requires a high-effort environment mapping. The latter approach, instead, is characterized by higher accuracy, but 
TABLE II

Comparison of Magnetic-Field Based Positioning Systems

\begin{tabular}{|c|c|c|c|c|c|c|}
\hline Reference & Accuracy & Cost & Power (or Current) & Operation Range & Complexity & Information \\
\hline Earth's Magnetic Field $74, \overline{77}-83$ & Room level up to $1 \mathrm{~m}$ & Low & Low & High & Medium & mostly $2 \mathrm{D}$ \\
\hline Blankenbach 75], 91 & $10-50 \mathrm{~cm}$ & Low to medium & $12 \mathrm{~A}$ & $15 \mathrm{~m}$ & medium & 3D \\
\hline Prigge [95] & centimeter level & low to medium & $\sim 100 \mathrm{~W}$ (estimated) & $<10 \mathrm{~m}$ & medium & $3 \mathrm{D}$ \\
\hline Sheinker 122-124 & $0.8 \mathrm{~m}(3 \mathrm{D})$ and $0.4 \mathrm{~m} \mathrm{(2D)}$ & medium to high & up to $8 \mathrm{~A}$ & up to $100 \mathrm{~m}$ & low to medium & $3 \mathrm{D}$ \\
\hline Carbone $7 \overline{76}, 1 \overline{129}, \overline{130}$ & $0.3 \mathrm{~m}$ & low & $0.02 \mathrm{~A}$ & $10-30 \mathrm{~m}$ & low & $2 \mathrm{D}$ \\
\hline Arumugam $[12 \overline{8} ; 69]$ & $0.12 \mathrm{~m} ; 0.77 \mathrm{~m}$ & medium to high & $0.56 \mathrm{~W}$ & $40 \mathrm{~m} ; 30 \mathrm{~m}$ & medium to high & $1 \mathrm{D} ; 5 \mathrm{D}$ \\
\hline Pirkl 126 & $4-40 \mathrm{~cm}$ & low to medium & $0.17 \mathrm{~A}$ & $4 \mathrm{~m}$ & medium to high & $3 \mathrm{D}$ \\
\hline MagLoc 127 & $0.5-0.8 \mathrm{~m}$ & low to medium & $0.1 \mathrm{~W}$ & - & medium & $3 \mathrm{D}$ \\
\hline Commercial 70], $144-147$ & millimeters to meters & high & - & meters to largely extended areas & low to very high & $1 D-6 D$ \\
\hline
\end{tabular}

has the main disadvantage of higher power consumption and shorter operating range. Using artificially generated magnetic fields, 1D - 6D positioning and orientation systems have been developed, with a performance accuracy varying from millimeter order to tens of decimeters order over operating ranges of the order of a meter or of the order of tens of meters, respectively. The systems diversity shows that currently none of the available solutions completely dominates the others and that the choice is mostly oriented by application requirements.

\section{REFERENCES}

[1] R. Barnes, J. Winterbottom, and M. Dawson, "Internet geolocation and location-based services," IEEE Communications Magazine, vol. 49, no. 4, pp. 102-108, 2011.

[2] J. Schiller and A. Voisard, Location Based Services. San Francisco, CA, USA: Morgan Kaufmann Publishers Inc., 2004.

[3] A. Roy, P. De, and N. Saxena, "Location-based social video sharing over next generation cellular networks," IEEE Communications Magazine, vol. 53, no. 10, pp. 136-143, 2015.

[4] A. Al-Fuqaha, M. Guizani, M. Mohammadi, M. Aledhari, and M. Ayyash, "Internet of things: A survey on enabling technologies, protocols, and applications," IEEE Communications Surveys Tutorials, vol. 17 , no. 4 , pp. 2347-2376, 2015.

[5] C. Perera, C. Liu, and S. Jayawardena, "The emerging internet of things marketplace from an industrial perspective: A survey," IEEE Transactions on Emerging Topics in Computing, vol. 3, no. 4, pp. 585598, 2015.

[6] S. Riazul Islam, D. Kwak, M. Humaun Kabir, M. Hossain, and K.S. Kwak, "The internet of things for health care: A comprehensive survey," IEEE Access, vol. 3, pp. 678-708, 2015.

[7] P. Barsocchi, S. Chessa, F. Furfari, and F. Potorti, "Evaluating ambient assisted living solutions: The localization competition," IEEE Pervasive Computing, vol. 12, no. 4, pp. 72-79, 2013.

[8] L. Palopoli, A. Argyros, J. Birchbauer, A. Colombo, D. Fontanelli, A. Legay, A. Garulli, A. Giannitrapani, D. Macii, F. Moro, P. Nazemzadeh, P. Padeleris, R. Passerone, G. Poier, D. Prattichizzo, T. Rizano, L. Rizzon, S. Scheggi, and S. Sedwards, "Navigation assistance and guidance of older adults across complex public spaces: the dali approach," Intelligent Service Robotics, vol. 8, no. 2, pp. 77-92, 2015.

[9] G. Sterling, Magnetic Positioning - The Arrival of 'Indoor GPS', Opus Research Report, Opus research Inc., San Francisco, 2014. [Online]. Available: http://opusresearch.net/ wordpress/pdfreports/MagneticPositioning_Opus_Jun2014.pdf

[10] Indoor Location Positioning Technology: Research, Solutions \& Trends. Grizzly Analytics, updated February 12, 2014.

[11] IZat. [Online]:. Qualcomm Inc. (Retrieved March 2015). [Online]. Available: http://www.qca.qualcomm.com/mobile-connectivity/ location/

[12] (2011) CSR debuts an indoor location and navigation breakthrough with SiRFstarV architecture and SiRFusion platform. CSR-Cambridge Silicon Solution. (Press Release). [Online]. Available: http://www.csr. com/news/pr/release/682/en

[13] "Eager: Collaborative research: Time critical localization in mobile networks," September 2013, granted by the National Science Foundation, award number 1441990 .
[14] "Whyphyloc8: Dynamic WiFi positioning using physical layer parameters for location-based services and security," 2012, granted by the National Science Foundation, award number 1232274. [Online]. Available: http://www.nsf.gov/awardsearch/showAward?AWD_ID=1232274

[15] "Entwicklung eines lokalen positionierungssystems fur den indoorbereich unter verwendung von knstlich erzeugten magnetfeldern (MILPS-2)," project funded by Deutsche Forschungsgemeinschaft: (German Research Foundation) (BL 1092/1-1).

[16] "BUTLER," project funded by European Union FP7. [Online]. Available: http://www.iot-butler.eu

[17] S. He and S.-H. Chan, "Wi-Fi fingerprint-based indoor positioning: Recent advances and comparisons," IEEE Communications Surveys Tutorials, 2015.

[18] Q. Vo and P. De, "A survey of fingerprint based outdoor localization," IEEE Communications Surveys Tutorials, 2015.

[19] H. Liu, H. Darabi, P. Banerjee, and J. Liu, "Survey of wireless indoor positioning techniques and systems," IEEE Transactions on Systems, Man, and Cybernetics, Part C: Applications and Reviews, vol. 37, no. 6, pp. 1067-1080, Nov 2007.

[20] R. Mautz, "Indoor positioning technologies," 2012, habilitation Thesis, ETH-Zurich. [Online]. Available: http://e-collection.library.ethz.ch/ eserv/eth:5659/eth-5659-01.pdf

[21] M. Erol-Kantarci, H. Mouftah, and S. Oktug, "A survey of architectures and localization techniques for underwater acoustic sensor networks," IEEE Communications Surveys Tutorials, vol. 13, no. 3, pp. 487-502, 2011.

[22] Y. Gu, A. Lo, and I. Niemegeers, "A survey of indoor positioning systems for wireless personal networks," IEEE Communications Surveys Tutorials, vol. 11, no. 1, pp. 13-32, 2009.

[23] R. Kulkarni, A. Forster, and G. Venayagamoorthy, "Computational intelligence in wireless sensor networks: A survey," IEEE Communications Surveys Tutorials, vol. 13, no. 1, pp. 68-96, 2011.

[24] I. Guvenc and C.-C. Chong, "A survey on TOA based wireless localization and NLOS mitigation techniques," IEEE Communications Surveys Tutorials, vol. 11, no. 3, pp. 107-124, 2009.

[25] R. Harle, "A survey of indoor inertial positioning systems for pedestrians," IEEE Communications Surveys Tutorials, vol. 15, no. 3, pp. 1281-1293, 2013

[26] P. Pathak, X. Feng, P. Hu, and P. Mohapatra, "Visible light communication, networking, and sensing: A survey, potential and challenges," IEEE Communications Surveys Tutorials, vol. 17, no. 4, pp. 20472077, 2015.

[27] L. Xie, Y. Yin, A. Vasilakos, and S. Lu, "Managing RFID data: Challenges, opportunities and solutions," IEEE Communications Surveys Tutorials, vol. 16, no. 3, pp. 1294-1311, 2014.

[28] Z. Sahinoglu, S. Gezici, and I. Güvenc, Ultra-wideband Positioning Systems: Theoretical Limits, Ranging Algorithms, and Protocols. Cambridge University Press, 2008.

[29] J. Schleppe and G. Lachapelle, "Tracking performance of a HSGPS receiver under avalanche deposited snow," GPS Solutions, vol. 12, no. 1, pp. 13-21, 2008.

[30] Z. Jiang and P. Groves, "NLOS GPS signal detection using a dualpolarisation antenna," GPS Solutions, pp. 1-12, 2012.

[31] O. Le Marchand, P. Bonnifait, J. Iba nez-Guzmán, D. Betaille, and F. Peyret, "Characterization of GPS multipath for passenger vehicles across urban environments," ATTI dell'Istituto Italiano di Navigazione, no. 189, pp. 77 - 88, Jul. 2009.

[32] S. Wang and M. Green, "Mobile location method for non-line-of-sight situation," in Proc. IEEE Vehic. Technol. Conf. (VTC), vol. 2, 2000 pp. 608-612.

[33] C. Yang and $\mathrm{H}$. rong Shao, "WiFi-based indoor positioning," IEEE Communications Magazine, vol. 53, no. 3, pp. 150-157, 2015. 
[34] W. Sun, J. Liu, C. Wu, Z. Yang, X. Zhang, and Y. Liu, "MoLoc: On distinguishing fingerprint twins," in IEEE 33rd International Conference on Distributed Computing Systems (ICDCS), 2013, pp. 226-235.

[35] T. Sathyan, D. Humphrey, and M. Hedley, "WASP: A system and algorithms for accurate radio localization using low-cost hardware," IEEE Transactions on Syst., Man, and Cybern., Part C (Applications and Reviews), vol. 41, no. 2, pp. 211-222, 2011.

[36] L. Luoh, "ZigBee-based intelligent indoor positioning system," Soft Computing, vol. 18, no. 3, pp. 443-456, March 2014.

[37] S. Schwarzer, M. Vossiek, M. Pichler, and A. Stelzer, "Precise distance measurement with IEEE 802.15.4 (ZigBee) devices," in IEEE Radio and Wireless Symposium, 2008, pp. 779-782.

[38] D. Capriglione, D. Casinelli, and L. Ferrigno, "Use of frequency diversity to improve the performance of RSSI-based distance measurements," in Measurements Networking (M N), 2015 IEEE International Workshop on, 2015, pp. 1-6.

[39] V. Pasku, M. L. Fravolini, and A. Moschitta, "Effects of antenna directivity on RF ranging when using space diversity techniques," Measurement, 2015.

[40] R. Faragher and R. Harle, "Location fingerprinting with Bluetooth Low Energy beacons," IEEE Journal on Selected Areas in Communications, vol. 33, no. 11, pp. 2418-2428, 2015.

[41] M. Ji, J. Kim, J. Jeon, and Y. Cho, "Analysis of positioning accuracy corresponding to the number of BLE beacons in indoor positioning system," in 17th International Conference on Advanced Communication Technology (ICACT), 2015, pp. 92-95.

[42] S. Gezici, Z. Tian, G. Giannakis, H. Kobayashi, A. Molisch, H. Poor, and Z. Sahinoglu, "Localization via ultra-wideband radios: A look at positioning aspects for future sensor networks," IEEE Signal Processing Magazine, vol. 22, no. 4, pp. 70-84, July 2005.

[43] P. Clarke. Impulse radio sets record for distance measurement [Online]:. (Retrieved 2016). [Online]. Available: http://www.eetimes. com/document.asp?doc_id=1280767

[44] B. Denis, J. Keignart, and N. Daniele, "Impact of NLOS propagation upon ranging precision in UWB systems," in Ultra Wideband Systems and Technologies, 2003 IEEE Conference on, 2003, pp. 379-383.

[45] Ranging radio. [Online]:. Time Domain Corporate. (Retrieved 2016). [Online]. Available: http://www.timedomain.com/

[46] Decawave. [Online]:. (Retrieved 2016). [Online]. Available: http: //www.decawave.com/

[47] Ubisense. [Online]:. Ubisense Group plc. St Andrew's House, St Andrew's Road, Chesterton, Cambridge, CB4 1DL. UK. (Retrieved 2016). [Online]. Available: http://ubisense.net/en

[48] Redpoint RTLS tags. [Online]: Redpoint Positioning Corporation (Retrieved 2016). [Online]. Available: http://www.redpointpositioning. $\mathrm{com} /$

[49] J. Reed, K. Krizman, B. Woerner, and T. Rappaport, "An overview of the challenges and progress in meeting the E-911 requirement for location service," Communications Magazine, IEEE, vol. 36, no. 4, pp 30-37, 1998

[50] A. Broumandan, T. Lin, J. Nielsen, and G. Lachapelle, "Practical results of hybrid AOA/TDOA geo-location estimation in CDMA wireless networks," in Vehicular Technology Conference, 2008. VTC 2008-Fall. IEEE 68th, 2008, pp. 1-5.

[51] M. Wylie-Green and S. Wang, "Robust range estimation in the presence of the non-line-of-sight error," in Vehicular Technology Conference, 2001. VTC 2001 Fall. IEEE VTS 54th, vol. 1, 2001, pp. 101-105 vol.1.

[52] A. Lazaro, D. Girbau, and D. Salinas, "Radio link budgets for UHF RFID on multipath environments," Antennas and Propagation, IEEE Transactions on, vol. 57, no. 4, pp. 1241-1251, 2009.

[53] A. Koutsou, F. Seco, A. Jimenez, J. Roa, J. Ealo, C. Prieto, and J. Guevara, "Preliminary localization results with an RFID based indoor guiding system," in Intelligent Signal Processing, 2007. WISP 2007. IEEE International Symposium on, 2007, pp. 1-6.

[54] F. juan Zhu, Z. heng Wei, B.-J. Hu, J. guang Chen, and Z. min Guo, "Analysis of indoor positioning approaches based on active RFID," in Wireless Communications, Networking and Mobile Computing, 2009. WiCom '09. 5th International Conference on, 2009, pp. 1-4.

[55] T. Bechteler and H. Yenigun, "2-D localization and identification based on SAW ID-tags at $2.5 \mathrm{GHz}$," Microwave Theory and Techniques, IEEE Transactions on, vol. 51, no. 5, pp. 1584 - 1590, may 2003.

[56] T. Hall, "Radiolocation using AM broadcast signals," Ph.D. Dissertation, Massachusetts Institute of Technology, 2002.

[57] J. McEllroy, "Navigation using signals of opportunity in the AM transmission band," Ph.D. Dissertation, Air Force Institute of Technology, 2006
[58] A. Lauri, F. Colone, R. Cardinali, C. Bongioanni, and P. Lombardo, "Analysis and emulation of FM radio signals for passive radar," in Aerospace Conference, 2007 IEEE, 2007, pp. 1-10.

[59] A. Tasdelen and H. Koymen, "Range resolution improvement in passive coherent location radar systems using multiple FM radio channels," IET Forum on Waveform Diversity and Design in Communications, Radar and Sonar, pp. 23-31(8), 2006.

[60] J. Farrell, Aided navigation: GPS with high rate sensors. McGrawHill, Inc., 2008.

[61] J.-O. Nilsson, I. Skog, and P. Handel, "Performance characterisation of foot-mounted ZUPT-aided INSs and other related systems," in International Conference on Indoor Positioning and Indoor Navigation (IPIN), 2010, pp. 1-7.

[62] J.-O. Nilsson, A. Gupta, and P. Handel, "Foot-mounted inertial navigation made easy," in International Conference on Indoor Positioning and Indoor Navigation (IPIN), 2014, pp. 24-29.

[63] A. De Angelis, A. Moschitta, P. Carbone, M. Calderini, S. Neri, R. Borgna, and M. Peppucci, "Design and characterization of a portable ultrasonic indoor 3-D positioning system," IEEE Transactions on Instrumentation and Measurement, vol. 64, no. 10, pp. 2616-2625, 2015.

[64] C. Medina, J. C. Segura, and A. De la Torre, "Ultrasound indoor positioning system based on a low-power wireless sensor network providing sub-centimeter accuracy," Sensors, vol. 13, no. 3, pp. 35013526, 2013.

[65] H. Hile and G. Borriello, "Positioning and orientation in indoor environments using camera phones," IEEE Computer Graphics and Applications, vol. 28, no. 4, pp. 32-39, 2008.

[66] K. Sjö, D. G. López, C. Paul, P. Jensfelt, and D. Kragic, "Object search and localization for an indoor mobile robot," Journal of Computing and Information Technology, vol. 17, no. 1, pp. 67-80, 2009

[67] B. Burki, S. Guillaume, P. Sorber, and H.-P. Oesch, "DAEDALUS: A versatile usable digital clip-on measuring system for total stations," in International Conference on Indoor Positioning and Indoor Navigation (IPIN), 2010, pp. 1-10.

[68] F. Boochs, R. Schutze, C. Simon, F. Marzani, H. Wirth, and J. Meier, "Increasing the accuracy of untaught robot positions by means of a multi-camera system," in International Conference on Indoor Positioning and Indoor Navigation (IPIN), 2010, pp. 1-9.

[69] D. Arumugam, J. Griffin, D. Stancil, and D. Ricketts, "Threedimensional position and orientation measurements using magnetoquasistatic fields and complex image theory [measurements corner]," IEEE Antennas and Propagation Magazine, vol. 56, no. 1, pp. 160173, Feb 2014.

[70] Fastrak motion tracking system. Polhemus Inc. (Retrieved April. 2016). [Online]. Available: http://polhemus.com/motion-tracking/all-trackers/ fastrak/

[71] I. Khalfin and R. Jones, "Methods and apparatus for electromagnetic position and orientation tracking with distortion compensation," US Patent US6 400139 B1, 1999.

[72] B. Li, T. Gallagher, A. G. Dempster, and C. Rizos, "How feasible is the use of magnetic field alone for indoor positioning?" in Proc. 2012 IEEE International Conference on Indoor Positioning and Indoor Navigation (IPIN), November 2012, pp. 13-15.

[73] A. Saxena and M. Zawodnjok, "Indoor positioning system using geo-magnetic field," in Proc. IEEE International Instrumentation and Measurement Technology Conference (I2MTC), 2014, pp. 572-577.

[74] J. Haverinen and A. Kemppainen, "A global self-localization technique utilizing local anomalies of the ambient magnetic field," in Proc. 2009 IEEE International Conference on Robotics and Automation, Kobe, Japan, May 2009, pp. 3142-3147.

[75] J. Blankenbach and A. Norrdine, "Position estimation using artificial generated magnetic fields," in Proc. of 2010 IEEE International Conference on Indoor Positioning and Indoor Navigation (IPIN), September 2010, pp. 1-5.

[76] G. D. Angelis, V. Pasku, A. D. Angelis, M. Dionigi, M. Mongiardo, A. Moschitta, and P. Carbone, "An indoor AC magnetic positioning system," IEEE Transactions on Instrumentation and Measurement, vol. 64 , no. 5, pp. 1267-1275, 2015.

[77] M. H. Afzal, V. Renaudin, and G. Lachapelle, "Use of earth's magnetic field for mitigating gyroscope errors regardless of magnetic perturbation," Sensors, vol. 11, pp. 11390-11414, 2011.

[78] B. Gozick, K. P. Subbu, R. Dantu, and T. Maeshiro, "Magnetic maps for indoor navigation," IEEE Transactions on Instrumentation and Measurement, vol. 60, no. 12, pp. 3883-3891, 2011. 
[79] J. Chung, M. Donahoe, C. Schmandt, I.-J. Kim, P. Razavai, and M. Wiseman, "Indoor location sensing using geo-magnetism," pp. 141154, 2011

[80] H. Xie, T. Gu, X. Tao, H. Ye, and J. Lu, "A reliability-augmented particle filter for magnetic fingerprinting based indoor localization on smartphone," IEEE Transactions on Mobile Computing, 2015.

[81] J. Jung, S. M. Lee, and H. Myung, "Indoor mobile robot localization and mapping based on ambient magnetic fields and aiding radio sources," IEEE Transactions on Instrumentation and Measurement, vol. 64, no. 7, pp. 1922-1934, 2015.

[82] Y. Shu, C. Bo, G. Shen, C. Zhao, L. Li, and F. Zhao, "Magicol: Indoor localization using pervasive magnetic field and opportunistic WiFi sensing," IEEE Journal on Selected Areas in Communications, vol. 33, no. 7, pp. 1443-1457, 2015.

[83] E. L. Grand and S. Thrun, "3-Axis magnetic field mapping and fusion for indoor localization," in 2012 IEEE Conference on Multisensor Fusion and Integration for Intelligent Systems (MFI), 2012, pp. 358364.

[84] J. Jackson, "Classical Electrodynamics," John Wiley and Sons Inc., 1999.

[85] C. A. Balanis, Antenna theory: analysis and design. John Wiley \& Sons, 2005

[86] S. I. Babic and C. Akyel, "Calculating mutual inductance between circular coils with inclined axes in air," IEEE Transactions on Magnetics, vol. 44, no. 7, pp. 1743-1750, July 2008.

[87] S. Babic, F. Sirois, C. Akyel, and C. Girardi, "Mutual inductance calculation between circular filaments arbitrarily positioned in space: Alternative to Grover's formula," IEEE Transactions on Magnetics, vol. 46, no. 9, pp. 3591-3600, Sept 2010.

[88] W. Yang, C. Hu, M. Li, M. Q. H. Meng, and S. Song, "A new tracking system for three magnetic objectives," IEEE Transactions on Magnetics, vol. 46, no. 12, pp. 4023-4029, 2010

[89] X. Wang, M. Q. H. Meng, and C. Hu, "A localization method using 3-axis magnetoresistive sensors for tracking of capsule endoscope," in 2006 28th Annual International Conference of the IEEE Engineering in Medicine and Biology Society, 2006, pp. 2522-2525.

[90] H. G. Kortier, J. Antonsson, H. M. Schepers, F. Gustafsson, and P. H. Veltink, "Hand pose estimation by fusion of inertial and magnetic sensing aided by a permanent magnet," IEEE Transactions on Neural Systems and Rehabilitation Engineering, vol. 23, no. 5, pp. 796-806, 2015.

[91] J. Blankenbach, A. Norrdine, and H. Hellmers, "A robust and precise 3D indoor positioning system for harsh environments," in Proc. of IEEE International Conference on Indoor Positioning and Indoor Navigation (IPIN), November 2012

[92] H. Hellmers, A. Norrdine, J. Blankenbach, and A. Eichhorn, "An IMU/magnetometer-based indoor positioning system using Kalman filtering," in 2013 International Conference on Indoor Positioning and Indoor Navigation (IPIN), 2013, pp. 1-9.

[93] H. Hellmers, A. Eichhorn, A. Norrdine, and J. Blankenbach, "Indoor localisation for wheeled platforms based on IMU and artificially generated magnetic field," in 2014 Ubiquitous Positioning Indoor Navigation and Location Based Service (UPINLBS), 2014, pp. 255-264.

[94] Z. Kasmi, A. Norrdine, and J. Blankenbach, "Towards a decentralized magnetic indoor positioning system," Sensors, vol. 15, no. 12, p. 29799 , 2015

[95] E. A. Prigge and J. P. How, "Signal architecture for a distributed magnetic local positioning system," IEEE Sensors Journal, vol. 4, no. 6, pp. 864-873, 2004.

[96] F. Raab, E. Blood, S. T., and J. H., "Magnetic Position and Orientation Tracking System," IEEE Trans. on Aerospace and Electrical Systems, vol. AES-15, no. 5, pp. 709-718, 1979

[97] F. Raab, "Quasi-static magnetic-field technique for determining position and orientation," IEEE Trans. on Geoscience and Remote Sensing, vol. GE-19, no. 4, pp. 235-243, October 1981.

[98] H. P. Kalmus, "A new guiding and tracking system," Aerospace and Navigational Electronics, IRE Transactions on, vol. ANE-9, no. 1, pp. 7 -10, march 1962.

[99] J. S. Bladen and A. P. Anderson, "Position location system," US Patent No. 5,913,820, June 221999.

[100] L. H. Rorden and T. C. Moore, "Method and apparatus employing received independent magnetic field components of a transmitted alternating magnetic field for determining location," US Patent No. 4,710,708, December 11987

[101] N. Amorai-Moriya, M. Itzkovich, and B. Spivak, "System for three dimensional positioning and tracking," US Patent No. 6,316,934, November 132001.
[102] A. Plotkin and E. Paperno, "3-D Magnetic Tracking of a Single Subminiature Coil with a Large 2-D Array of Uniaxial Transmitters," in IEEE Trans. Magnetics, vol. 39, no. 5, Sept. 2003, pp. 3295-3297.

[103] M. Schneider, "Measuring position and orientation using magnetic fields," US Patent No. 6,073,043, June 62000.

[104] A. Govari, "Electromagnetic position single axis system," US Patent No. 6,484,118, Nov. 192002.

[105] E. Stathopoulos, V. Schlageter, B. Meyrat, Y. de Ribaupierre, and P. Kucera, "Magnetic pill tracking: a novel non-invasive tool for investigation of human digestive motility," Neurogastroenterology \& Motility, vol. 17, no. 1, pp. 148-154, 2005.

[106] S. Yabukami, H. Kanetaka, N. Tsuji, A. Itagaki, M. Yamaguchi, K. Arai, and H. Mitani, "A new tracking system of jaw movement using two magnets," in Magnetics Conference, 2002. INTERMAG Europe 2002. Digest of Technical Papers. 2002 IEEE International, 2002, pp. FV8-.

[107] X. Wu, W. Hou, C. Peng, X. Zheng, X. Fang, and J. He, "Wearable magnetic locating and tracking system for MEMS medical capsule," Sensors and Actuators A: Physical, vol. 141, no. 2, pp. 432 - 439 , 2008.

[108] C. Hu, M. Li, S. Song, W. Yang, R. Zhang, and M.-H. Meng, "A cubic 3-axis magnetic sensor array for wirelessly tracking magnet position and orientation," Sensors Journal, IEEE, vol. 10, no. 5, pp. 903-913, 2010.

[109] J. Sherman, J. Lubkert, R. Popovic, and M. DiSilvestro, "Characterization of a novel magnetic tracking system," Magnetics, IEEE Transactions on, vol. 43, no. 6, pp. 2725-2727, 2007.

[110] S. Hashi, Y. Tokunaga, S. Yabukami, M. Toyoda, K. Ishiyama, Y. Okazaki, and K. Arai, "Development of real-time and highly accurate wireless motion capture system utilizing soft magnetic core,' Magnetics, IEEE Transactions on, vol. 41, no. 10, pp. 4191-4193, 2005.

[111] C. Hu, S. Song, X. Wang, M. Q. H. Meng, and B. Li, "A novel positioning and orientation system based on three-axis magnetic coils," IEEE Transactions on Magnetics, vol. 48, no. 7, pp. 2211-2219, 2012.

[112] S. Song, W. Qiao, B. Li, C. Hu, H. Ren, and M. Q. H. Meng, "An efficient magnetic tracking method using uniaxial sensing coil," IEEE Transactions on Magnetics, vol. 50, no. 1, pp. 1-7, 2014.

[113] W. F. Loke, T. Y. Choi, T. Maleki, L. Papiez, B. Ziaie, and B. Jung, "Magnetic tracking system for radiation therapy," IEEE Transactions on Biomedical Circuits and Systems, vol. 4, no. 4, pp. 223-231, 2010.

[114] D. Roetenberg, P. J. Slycke, and P. H. Veltink, "Ambulatory position and orientation tracking fusing magnetic and inertial sensing," IEEE Transactions on Biomedical Engineering, vol. 54, no. 5, pp. 883-890, 2007.

[115] G. Pirkl, K. Stockinger, K. Kunze, and P. Lukowicz, "Adapting magnetic resonant coupling based relative positioning technology for wearable activitiy recogniton," in 2008 12th IEEE International Symposium on Wearable Computers, ISWC 2008., 2008, pp. 47-54.

[116] D. D. Arumugam, "Single-anchor 2-D magnetoquasistatic position sensing for short to long ranges above ground," IEEE Antennas and Wireless Propagation Letters, vol. 15, pp. 1325-1328, 2016.

[117] D. D. Arumugam and D. S. Ricketts, "Passive magnetoquasistatic position measurement using coupled magnetic resonances," IEEE Antennas and Wireless Propagation Letters, vol. 12, pp. 539-542, 2013.

[118] M. Reynolds, "Low frequency indoor radiolocation," Ph.D. Dissertation, Media Arts and Sciences, MIT, 2003.

[119] H. Schantz, "Near field phase behavior," IEEE International Symposium APS., vol. 3B, pp. 134-137, July 2005.

[120] — "Near field propagation law and a novel fundamental limit to antenna gain versus size," IEEE International Symposium APS. vol. 3A, pp. 237-240, July 2005.

[121] H. G. Schantz, "A real-time location system using near-field electromagnetic ranging," June 2007, pp. 3792-3795.

[122] A. Sheinker, B. Ginzburg, N. Salomonski, L. Frumkis, and B.-Z. Kaplan, "Localization in 2D using beacons of low frequency magnetic field," IEEE Journal of Selected Topics in Applied Earth Observations and Remote Sensing, vol. 6, no. 2, pp. 1020-1030, 2013.

[123] — , "Localization in 3-D using beacons of low frequency magnetic field," IEEE Transactions on Instrumentation and Measurement, vol. 62 , no. 12 , pp. 3194-3201, 2013.

[124] _ - "Remote tracking of a magnetic receiver using low frequency beacons," Measurement Science and Technology, vol. 25, no. 10, p. 105101, 2014

[125] A. Sheinker, B. Ginzburg, N. Salomonski, L. Frumkis, B.-Z. Kaplan, and M. B. Moldwin, "A method for indoor navigation based on mag- 
netic beacons using smartphones and tablets," Measurement, vol. 81, pp. $197-209,2016$.

[126] G. Pirkl and P. Lukowicz, "Robust, low cost indoor positioning using magnetic resonant coupling," in Proceedings of the 2012 ACM Conference on Ubiquitous Computing, ser. UbiComp '12, 2012, pp. 431-440.

[127] T. E. Abrudan, Z. Xiao, A. Markham, and N. Trigoni, "Distortion rejecting magneto-inductive three-dimensional localization (MagLoc)," IEEE Journal on Selected Areas in Communications, vol. 33, no. 11, pp. 2404-2417, 2015.

[128] D. D. Arumugam, J. D. Griffin, and D. D. Stancil, "Experimental demonstration of complex image theory and application to position measurement," IEEE Antennas and Wireless Propagation Letters, vol. 10, pp. 282-285, 2011.

[129] V. Pasku, A. D. Angelis, M. Dionigi, G. D. Angelis, A. Moschitta, and P. Carbone, "A positioning system based on low-frequency magnetic fields," IEEE Transactions on Industrial Electronics, vol. 63, no. 4, pp. 2457-2468, 2016.

[130] G. D. Angelis, A. D. Angelis, V. Pasku, A. Moschitta, and P. Carbone, "An experimental system for tightly coupled integration of GPS and AC magnetic positioning," IEEE Transactions on Instrumentation and Measurement, vol. 65, no. 5, pp. 1232-1241, 2016.

[131] V. Pasku, A. D. Angelis, A. Moschitta, P. Carbone, J. O. Nilsson, S. Dwivedi, and P. Haendel, "A magnetic ranging-aided dead-reckoning positioning system for pedestrian applications," IEEE Transactions on Instrumentation and Measurement, 2017.

[132] R. Olsen and A. Farstad, "Electromagnetic direction finding experiments for location of trapped miners," Geoscience Electronics, IEEE Transactions on, no. 4, pp. $178-185$, oct. 1973.

[133] J. R. Wait, "Electromagnetic induction technique for locating a buried source," Geoscience Electronics, IEEE Transactions on, vol. 9, no. 2, pp. 95-98, 1971.

[134] J. Wait, "Criteria for locating an oscillating magnetic dipole buried in the earth," Proceedings of the IEEE, vol. 59, no. 6, pp. 1033-1035, 1971.

[135] T. W. H. Caffey and L. Romero, "Locating a buried magnetic dipole," Geoscience and Remote Sensing, IEEE Transactions on, vol. GE-20, no. 2, pp. 188-192, 1982.

[136] A. Markham, N. Trigoni, D. W. Macdonald, and S. A. Ellwood, "Underground localization in 3-D using magneto-inductive tracking," IEEE Sensors Journal, vol. 12, no. 6, pp. 1809-1816, 2012.

[137] N. Ayuso, J. Cuchi, F. Lera, and J. Villarroel, "Avalanche beacon magnetic field calculations for rescue techniques improvement," in Geoscience and Remote Sensing Symposium, 2007. IGARSS 2007. IEEE International, 2007, pp. 722-725.

[138] P. Pinies and J. Tardos, "Fast localization of avalanche victims using sum of gaussians," in Robotics and Automation, 2006. ICRA 2006. Proceedings 2006 IEEE International Conference on, 2006, pp. 3989 3994.

[139] R. Sharpe, "Systems for locating and identifying victims of manmade or natural disasters," US Patent No. 2006/0148423 A1, July 62006.

[140] J. Schweizer and G. Krusi, "Testing the performance of avalanche transceivers," Cold Regions Science and Technology, vol. 37, no. 3, pp. $429-438,2003$.

[141] P. Holler and F. Gibler, "On the practical use of avalanche beacons; the austrian transceiver test 2001," International Snow Science Workshop, pp. 1-5, 2002.

[142] J. Schweizer, M. Genswein, F. Jarry, and D. Letang, "Transceiver performance when searching for multiple burials," International Snow Science Workshop, pp. 341-347, 2012.

[143] F. Shubitidze, A. Bijamov, G. Schultz, J. Miller, and I. Shamatava, "Investigating tx coils and magnetic field $\mathrm{rx}$ sensor configurations for underwater geo-location," in SPIE Defense, Security, and Sensing. International Society for Optics and Photonics, 2011, pp. $80170 \mathrm{~V}-$ $80170 \mathrm{~V}$.

[144] Q-track real time location systems. Q-track Corp. (Retrieved November 2014). [Online]. Available: http://www.q-track.com

[145] trakSTAR and driveBAY. Ascension Technology Corporation. (Retrieved April. 2016). [Online]. Available: http://www. ascension-tech.com/

[146] STEM system and razer hydra. Sixense Entertainment, Inc. (Retrieved April. 2016). [Online]. Available: http://sixense.com/

[147] Indooratlas. IndoorAtlas Ltd. (Retrieved April. 2016). [Online]. Available: https://www.indooratlas.com/

[148] (2013) New indoor navigation technologies work where GPS can't. IEEE Spectrum Magazine. [On- line]: [Online]. Available: http://spectrum.ieee.org/telecom/wireless/ new-indoor-navigation-technologies-work-where-gps-cant 